\title{
Closing the Loop
}

\author{
Revital Nimri ${ }^{1}$, Pearl Audon ${ }^{2}$, Jordan E. Pinsker ${ }^{2}$, and Eyal Dassau ${ }^{2,3}$
}

\section{Introduction}

$\mathbf{T}$

ECHNOlOgicAl ADVANCEMENTS ARE rapidly changing the lifestyle and healthcare of people with type 1 diabetes mellitus (T1DM). Current open-loop devices such as continuous glucose monitors and insulin pumps require constant human monitoring of insulin delivery. Moreover, the lives of people with T1DM are dynamic, with changing insulin requirements due to exercise, meals, and other activities. Inaccurate insulin dosing can lead to hypo- or hyperglycemia. Thus, the potential for an automated insulin delivery system (closed-loop or artificial pancreas [AP]) to minimize the work needed to achieve ideal insulin dosing is a potential seismic shift in diabetes care. An AP system merges together an insulin pump, continuous glucose monitor, and a computerized control algorithm to regulate insulin delivery in response to real-time sensor data and future predicted glucose values. The ultimate goal is to create a "hands-off" approach to insulin delivery to minimize the burden of blood glucose management.

Rapid progress has been made over the past decade with development of the AP (closed-loop system or automated insulin delivery). Several academic groups worldwide developed and tested closed-loop systems that are either single hormone (modulation of insulin delivery alone) or dual hormone using insulin and glucagon. The clinical studies provided the evidence that closed-loop systems can improve glycemic control, reduce rates of hypoglycemia and hyperglycemia, improve A1c, and improve some aspects of diabetes management burden. These clinical studies provided solid evidence for the safety and efficacy of the closed-loop system to become an integral part of diabetes management, paving the way for pivotal studies for commercialization of the artificial pancreas.

The year 2017 marked a landmark achievement for closed-loop systems-U.S. Food and Drug Administration (FDA) approval of the first commercially available closed-loop device, the Medtronic 670G with Guardian 3 sensor, in the United States. In addition, clinical trials have progressed from the structured inpatient environment to unsupervised 24/7 real-world use. Many of the trials have moved beyond feasibility and are now of randomized crossover design. Studies have advanced to work to improve on different challenges and have included different age groups (pediatrics, adolescents, adults), physical activity (1), and different strategies for meal boluses. In addition, the state of dual-hormone systems, which add glucagon as either part of the integrated algorithm or as a rescue dose system, has also advanced. To increase speed of insulin action, intraperitoneal (IP) insulin delivery was also studied (2). Additionally, AP has been tested in the inpatient postoperative setting (3-5), the first publication on outpatient use of AP during pregnancy was published this year (6), and numerous studies are assessing the usability and acceptance of AP devices $(7,8)$. As research continues to improve upon the limitations of AP systems, this year's highlights have demonstrated that we are one step closer to fully closing the loop for people with diabetes.

\footnotetext{
${ }^{1}$ Jesse Z and Sara Lea Shafer Institute for Endocrinology and Diabetes, National Center for Childhood Diabetes, Schneider Children's Medical Center of Israel, Petah Tikva, Israel.

${ }^{2}$ Sansum Diabetes Research Institute, Santa Barbara, California.

${ }^{3}$ Harvard John A. Paulson School of Engineering and Applied Sciences, Harvard University, Cambridge, Massachusetts.
} 
Key Articles Reviewed for the Article

Safety of a hybrid closed-loop insulin delivery system in patients with type 1 diabetes

Bergenstal RM, Garg S, Weinzimer SA, Buckingham BA, Bode BW, Tamborlane $W V$, Kaufman FR

JAMA 2016; 316: 1407-1408

Glucose outcomes with the in-home use of a hybrid closed-loop insulin delivery system in adolescents and adults with type 1 diabetes

Garg SK, Weinzimer SA, Tamborlane WV, Buckingham BA, Bode BW, Bailey TS, Brazg RL, Ilany J, Slover RH, Anderson SM, Bergenstal RM, Grosman B, Roy A, Cordero TL, Shin J, Lee SW, Kaufman FR

Diabetes Technol Ther 2017; 19: 155-163

Home use of a bihormonal bionic pancreas versus insulin pump therapy in adults with type 1 diabetes: a multicentre randomised crossover trial

El-Khatib FH, Balliro C, Hillard MA, Magyar KL, Ekhlaspour L, Sinha M, Mondesir D, Esmaeili A, Hartigan H, Thompson MJ, Malkani S, Lock JP, Harlan DM, Clinton P, Frank E, Wilson DM, DeSalvo D, Norlander L, Ly T, Buckingham BA, Diner J, Dezube M, Young LA, Goley A, Kirkman MS, Buse JB, Zhenn H, Selagamsetty RR, Damiano ER, Russell SJ

Lancet 2016; 389: 369-380

A simplified semiquantitative meal bolus strategy combined with single- and dual-hormone closed-loop delivery in patients with type 1 diabetes: a pilot study

Gingras V, Haidar A, Messier V, Legault L, Ladouceur M, Rabasa-Lhorert $R$

Diabetes Technol Ther 2016; 18: 464-471

Randomized trial of a dual-hormone artificial pancreas with dosing adjustment during exercise compared with no adjustment and sensor-augmented pump therapy

Jacobs PG, El Youssef J, Reddy R, Resalat N, Branigan D, Condon J, Preiser N, Ramsey K, Jones M, Edwards C, Kuehl K, Leitschuh J, Rajhbeharrysingh U, Castle JR

Diabetes Obes Metab 2016; 18: 1110-1119

Efficacy of single-hormone and dual-hormone artificial pancreas during continuous and interval exercise in adult patients with type 1 diabetes: randomised controlled crossover trial

Taleb N, Emami A, Suppere C, Messier V, Legault L, Ladouceur M, Chiasson J-L, Haidar A, Rabasa-Lhoret $R$

Diabetologia 2016; 59: 2561-2571

Heart rate informed artificial pancreas system enhances glycemic control during exercise in adolescents with T1DM

DeBoer MD, Cherñavvsky DR, Topchyan K, Kovatchev BP, Francis GL, Breton $M D$

Pediatric Diabetes 2017; 18: 540-546

Closed-loop insulin delivery for adults with type 1 diabetes undertaking high-intensity interval exercise versus moderate-intensity exercise:

a randomized, crossover study

Jayawardene DC, McAuley SA, Horsburgh JC, Gerche AL, Jenkins AJ, Ward GM, MacIsaac RJ, Roberts TJ, Grosman B, Kurtz N, Roy A, O'Neal DN

Diabetes Technol Ther 2017; 19: 340-348 
Closed-loop insulin delivery in inpatients with type 2 diabetes: a randomised, parallel-group trial

Thabit H, Hartnell S, Allen JM, Lake A, Wilinska ME, Ruan Y, Evans $M L$, Coll AP, Hovorka $R$

Lancet Diabetes Endocrinol 2017; 5: 117-124

Closed-loop insulin delivery during pregnancy in women with type 1 diabetes

Stewart ZA, Wilinska ME, Hartnell S, Temple RC, Rayman $G$, Stanley KP,

Simmons D, Law GR, Scott EM, Hovorka R, Murphy HR

N Engl J Med 2016; 375: 644-654

Intraperitoneal insulin delivery provides superior glycemic regulation to subcutaneous insulin delivery in model predictive control-based fully-automated artificial pancreas in patients with type 1 diabetes: a pilot study

Dassau E, Renard E, Place J, Farret A, Pelletier M-J, Lee J, Huyett LM,

Chakrabarty A, Doyle FJ III, Zisser HC

Diabetes Obes Metab 2017; 19: 1698-1705

Day-and-night glycaemic control with closed-loop insulin delivery versus conventional insulin pump therapy in free-living adults with well controlled type 1 diabetes: an open-label, randomised, crossover study

Bally L, Thabit H, Kojzar H, Mader JK, Qerimi-Hyseni J, Hartnell S, Tauschmann M, Allen JM, Wilinska ME, Pieber TR, Evans ML, Hovorka $R$

Lancet Diabetes Endocrinol 2017; 5: 261-270

Application of zone model predictive control artificial pancreas during extended use of infusion set and sensor: a randomized crossover-controlled home-use trial

Forlenza GP, Deshpande S, Ly TT, Howsmon DP, Cameron F, Baysal $N$, Mauritzen E, Marcal T, Towers L, Bequette W, Huyett LM, Pinsker JE, Gondhalekar R, Doyle FJ III, Maahs DM, Buckingham BA, Dassau E

Diabetes Care 2017; 40: 1096-1102

MD-logic overnight type 1 diabetes control in home settings: a multicentre, multinational, single blind randomized trial

Nimri $R$, Bratina B, Kordonouri $O$, Avbelj Stefanija M, Fath M, Biester T, Muller I, Atlas E, Miller S, Fogel A, Phillip M, Danne T, Battelino T

Diabetes Obes and Metab 2017; 19: 553-561

Home use of day-and-night hybrid closed-loop insulin delivery in suboptimally controlled adolescents with type 1 diabetes: a 3-week, free-living, randomized crossover trial

Tauschmann M, Allen JM, Wilinska ME, Thabit H, Acerini CL, Dunger DB, Hovorka $R$ Diabetes Care 2016; 39: 2019-2025

\section{PIVOTAL STUDIES}

This year marked the first FDA approval of a closed-loop commercial product, the Medtronic 670G system. In the manuscripts listed below, Bergenstal et al. and Garg et al. discuss the clinical trial that led to FDA approval of the first hybrid closed-loop commercial product. These manuscripts are described together in one synopsis.

\section{Safety of a hybrid closed-loop insulin delivery system in patients with type 1 diabetes}

Bergenstal $R M^{1}$, Garg $S^{2}$, Weinzimer $S A^{3}$, Buckingham $B A^{4}$, Bode $B W^{5}$, Tamborlane $W V^{3}$, Kaufman $F R^{6}$
${ }^{1}$ International Diabetes Center, Minneapolis, $M N ;{ }^{2}$ Barbara Davis Center for Diabetes, University of Colorado Denver, Aurora, CO; ${ }^{3}$ Yale University, New Haven, CT; ${ }^{4}$ Stanford University, Stanford, CA; ${ }^{5}$ Atlanta Diabetes Associates, Atlanta, GA; ${ }^{6}$ Medtronic, Northridge, CA

JAMA 2016; 316: 1407-1408

Glucose outcomes with the in-home use of a hybrid closed-loop insulin delivery system in adolescents and adults with type 1 diabetes

Garg $S K^{1}$, Weinzimer $S A^{2}$, Tamborlane $W V^{2}$, Buckingham $B A^{3}$, Bode $B W^{4}$, Bailey $T S^{5}$, Brazg $R L^{6}$, 
Ilany $J^{7}$, Slover $R H^{1}$, Anderson $S M^{8}$, Bergenstal $R M^{9}$, Grosman $B^{10}$, Roy $A^{10}$, Cordero $T L^{10}$, Shin $J^{10}$, Lee $S W^{10}$, Kaufman $\mathrm{FR}^{10}$

${ }^{1}$ Barbara Davis Center for Diabetes, University of Colorado Denver, Aurora, CO; ${ }^{2}$ Yale University, New Haven, CT; ${ }^{3}$ Stanford University, Stanford, CA; ${ }^{4}$ Atlanta Diabetes Associates, Atlanta, GA; ${ }^{5}$ AMCR Institute, Escondido, CA; ${ }^{6}$ Rainier Clinical Research Center, Renton, WA; ${ }^{7}$ Sheba Medical Center, Tel Hashomer, Israel; ${ }^{8}$ University of Virginia, Charlottesville, VA; ${ }^{9}$ International Diabetes Center, Minneapolis, $\mathrm{MN} ;{ }^{10}$ Medtronic, Northridge, CA

Diabetes Technol Ther 2017; 19: 155-163

\section{Background}

The purpose of this study was to investigate the safety and efficacy of in-home use of the Medtronic MiniMed 670G hybrid closed-loop (HCL) system.

\section{Methods}

Adolescents $(n=30)$ and adults $(n=94)$ with type 1 diabetes participated in a multicenter pivotal trial. The Medtronic MiniMed 670G system was used during a 2-week runin phase in open-loop mode. Then, auto mode was enabled during a 3-month study phase. A 6-day hotel stay that included one day of frequent blood sample testing with a reference measurement (i-STAT) occurred with use of auto mode.

\section{Results}

From baseline run in to the end of study, adolescent glycated hemoglobin $(\mathrm{HbA} 1 \mathrm{c})$ levels decreased from $7.7 \pm 0.8 \%$ to $7.1 \pm 0.6 \%$ and adult HbA1c levels decreased from $7.3 \pm 0.9 \%$ to $6.8 \pm 0.6 \% \quad(P<0.001)$. The proportion of overall in-target $(71-180 \mathrm{mg} / \mathrm{dL})$ sensor glucose (SG) values increased from $60.4 \pm 10.9 \%$ to $67.2 \pm 8.2 \%(P<0.001)$ in adolescents and from $68.8 \pm 11.9 \%$ to $73.8 \pm 8.4 \%$ in adults $(P<0.001)$. Overall sensor accuracy, as measured by the mean absolute relative difference (MARD) \pm SD between SG and i-STAT venous blood glucose values during the hotel stay was $11.2 \pm 9.7 \%$ for adolescents and $10.0 \pm 8.7 \%$ for adults. No severe hypoglycemic or diabetic ketoacidosis events were observed.

\section{Conclusion}

Home use of the HCL system was found to be safe and effective in controlling blood glucose levels for adolescents and adults. Furthermore, the intervention phase demonstrated increased time in target, with reductions in hyperglycemia, hypoglycemia, and $\mathrm{HbA} 1 \mathrm{c}$ as compared with the baseline run-in period.

\section{Comments}

The Medtronic 670G is a single-hormone HCL system that adjusts basal insulin delivery in response to sensor glucose levels. The control algorithm is integrated into the pump. The pivotal study results demonstrated the safety of the Medtronic $670 \mathrm{G}$ to control blood glucose levels for people with T1DM with no significant adverse events. This pivotal study design was to evaluate the safety of the system for regulatory purposes; therefore, no control group was used and efficacy data are limited. Although the study was single-arm and nonrandomized by design, the improvement in glycemic variability and HbA1c levels from baseline were significant. Improvement of these outcomes will no doubt lead to decreased long-term diabetes-related complications. This study led to FDA approval of the Medtronic 670G for commercial sale beginning in the United States in 2017. Future outpatient studies are planned to examine results from long-term use of the HCL system.

\section{DUAL-HORMONE STUDIES}

\section{Home use of a bihormonal bionic pancreas versus insulin pump therapy in adults with type 1 diabetes: a multicentre randomised crossover trial}

El-Khatib $\mathrm{FH}^{1}$, Balliro $\mathrm{C}^{2}$, Hillard $\mathrm{MA}^{2}$, Magyar $\mathrm{KL}^{2}$, Ekhlaspour $L^{2}$, Sinha $M^{2}$, Mondesir $D^{2}$, Esmaeili $A^{2}$, Hartigan $\mathrm{H}^{4}$, Thompson $\mathrm{MJ}^{4}$, Malkani $\mathrm{S}^{4}$, Lock $\mathrm{JP}^{4}$, Harlan $D M^{4}$, Clinton $P^{5}$, Frank $E^{5}$, Wilson $D M^{5}$, DeSalvo $D^{5}$, Norlander $L^{5}$, Ly $T^{5}$, Buckingham $B A^{5}$, Diner $J^{6}$, Dezube $M^{6}$, Young $L A^{6}$, Goley $A^{6}$, Kirkman $M S^{6}$, Buse JB ${ }^{6}$, Zhenn $H^{3}$, Selagamsetty $R R^{l}$, Damiano $E R^{1}$, Russell $S J^{2}$

${ }^{1}$ Department of Biomedical Engineering, Boston University, Boston, MA; ${ }^{2}$ Diabetes Unit and Department of Medicine and ${ }^{3}$ Biostatistics Center, Massachusetts General Hospital, Boston, MA; ${ }^{4}$ Center for Clinical and Translational Science and the Diabetes Center of Excellence, University of Massachusetts Medical School, Worcester, MA; ${ }^{5}$ Division of Pediatric Endocrinology and Diabetes, Stanford University School of Medicine, Palo Alto, CA; ${ }^{6}$ Diabetes Care Center, University of North Carolina at Chapel Hill School of Medicine, Chapel Hill, NC

Lancet 2016; 389: 369-380

\section{Background}

Use of a bihormonal (insulin and glucagon) artificial pancreas in a free-living, home-use setting has not been extensively studied. This study in patients with type 1 diabetes aimed to assess whether bihormonal bionic pancreas initialized only with body mass can safely reduce glucose levels and prevent hypoglycemia for participants living at home and engaging in daily activities without dietary or exercise restrictions.

\section{Methods}

A randomized crossover study was conducted including 43 adolescents and adults with T1DM. Twenty participants were assigned to bionic pancreas first and 19 were assigned to the insulin pump therapy first. The bionic pancreas was initialized with only the participant's body mass and had additional features to simplify meal boluses. The user interface allowed optional meal announcements, designated as "breakfast," "lunch," or "dinner," and of size "typical," "more than typical," "less than typical," or "a small bite." It was suggested, but not required, that patients use this feature 
immediately before eating the main meals of the day, but not snacks. Each arm of the study was 11 days in length, and patients continued with daily activities (i.e., exercise, driving). Autonomously adaptive dosing algorithms used data from the continuous glucose monitor (CGM) to control subcutaneous delivery of insulin and glucagon. The mean glucose concentration and time with CGM glucose concentration less than $3.3 \mathrm{mmol} / \mathrm{L}$ were analyzed over days $2-11$ in participants who completed both arms of the study.

\section{Results}

The mean CGM glucose concentration was significantly lower in the bionic pancreas period $7.8 \pm 0.6 \mathrm{mmol} / \mathrm{L}$ compared with $9.0 \pm 1.6 \mathrm{mmol} / \mathrm{L}$ in the comparator period $(P<0.0001)$. The average time with $\mathrm{CGM}$ glucose concentration less than $3.3 \mathrm{mmol} / \mathrm{L}$ was significantly lower in the bionic pancreas period $(0.6 \pm 0.6 \%)$ compared with $1.9 \pm 1.7 \%$ in the comparator period $(P<0.0001)$. The mean nausea score on the Visual Analogue Scale (scale 0-10) was significantly greater during the bionic pancreas period $(0.52 \pm 0.83)$ than in the comparator period $(0.05 \pm 0.17),(P=0.0024)$. No serious adverse events occurred during the bionic pancreas period of the study.

\section{Conclusion}

With the bihormonal bionic pancreas use, less time was spent in hypoglycemia, and lower mean blood glucose level was recorded compared with insulin pump therapy. The bihormonal bionic pancreas was found to be safe for use in a home setting without restrictions.

\section{Comments}

A fully automated bihormonal bionic pancreas will no doubt improve glycemic control in people with T1DM. Since only body weight was required to initialize the bihormonal bionic pancreas and carbohydrate counting was optional, less effort was required from both patients and physicians. Elimination of burdensome activities such as carefully calculating meal bolus doses will be a major advancement in diabetes care. However, additional work needs to be done to mitigate risk of being dependent on glucagon, and on side effects such as nausea. Also, additional information on the controller design to fit a singlehormone strategy is needed to evaluate an equitable comparison between dual- and single-hormone studies, as these results are comparable to single-hormone studies by other groups that suggest dual-hormone systems may not be significantly better than single-hormone systems (9). Additional long-term study is needed to determine how best to achieve ideal postprandial control while simplifying meal boluses in dual-hormone systems.

\section{A simplified semiquantitative meal bolus strategy combined with single- and dual-hormone closed-loop delivery in patients with type 1 diabetes: a pilot study}

Gingras $V^{1,2}$, Haidar $A^{1,3}$, Messier $V^{1}$, Legault $L^{4}$, Ladouceur $M^{5}$, Rabasa-Lhorert $R^{1-3,5,6}$
${ }^{1}$ Institut de Recherches Cliniques de Montréal, Montreal, Canada; ${ }^{2}$ Department of Nutrition, Université de Montréal, Montreal, Canada; ${ }^{3}$ Division of Experimental Medicine, McGill University, Montreal, Canada; ${ }^{4}$ Montreal Children's Hospital, McGill University Health Center, Montreal, Canada; ${ }^{5}$ Research Center of the Université de Montréal Hospital Center, Montreal, Canada; ${ }^{6}$ Montreal Diabetes Research Center, Montreal, Canada

Diabetes Technol Ther 2016; 18: 464-471

\section{Background}

Single- or dual-hormonal closed-loop system may reduce glycemic variability in patients with T1DM. Previous studies have attempted to simplify carbohydrate counting in closedloop systems (10); however, patients can have improved glucose control if they integrate meal boluses, even in a simplified form, alongside the use of closed-loop control (CLC).

\section{Methods}

A randomized outpatient trial was conducted with 12 adult participants with T1DM. A single-hormone closed-loop system group was used with carbohydrate content-matched boluses or with a simplified meal bolus strategy, and a dualhormone closed-loop system group was used with carbohydrate content-matched boluses or with a simplified meal bolus strategy. In the simplified strategy, participants selected a semiquantitative meal carbohydrate content size: snack, regular, large, or very large meal. Each patient also underwent sensor-augmented pump therapy. Mean sensor glucose level over a daytime (15-h) period was the primary outcome.

\section{Results}

Similar mean sensor glucose levels $(15 \mathrm{~h})$ were observed with the carbohydrate-matched boluses and with the simplified strategy using a single-hormone system (median [interquartile range]: 7.6 [7.2-8.1] vs. 8.0 [7.0-8.6] mmol/L; $P=0.90)$ and dual-hormone closed-loop systems (7.6 [6.7$9.1]$ vs. 7.0 [6.4-8.2] mmol/L; $P=0.08)$. Follow-up analyses showed that compared with sensor-augmented pump therapy, time spent in hypoglycemia increased with the simplified strategy but not with the carbohydrate-matched boluses.

\section{Conclusion}

Although the simplified strategy in this pilot study may result in higher risk for hypoglycemia, this strategy could potentially reduce the carbohydrate-counting burden in patients with T1DM while generally maintaining adequate glucose control.

\section{Comments}

This study is vital to assessing the type of meal bolus strategies applicable in pairing with single- or dualhormone closed-loop systems. Although the research points to an increased risk for hypoglycemia with the simplified meal bolus strategy, longer and larger outpatient studies are needed to increase power. A strength of the study was that most of the participants found little to no difficulty in the meal bolus strategy, which is key for adoption and continued long-term use of AP systems. 


\section{EXERCISE STUDIES}

\section{Randomized trial of a dual-hormone artificial pancreas with dosing adjustment during exercise compared with no adjustment and sensor-augmented pump therapy}

Jacobs $P G^{1}$, El Youssef $J^{2}$, Reddy $R^{1}$, Resalat $N^{1}$, Branigan $D^{2}$, Condon $\mathrm{J}^{1}$, Preiser $\mathrm{N}^{1}$, Ramsey $\mathrm{K}^{3}$, Jones $M^{2}$, Edwards $C^{2}$, Kuehl $K^{4}$, Leitschuh $\mathrm{J}^{1}$, Rajhbeharrysingh $U^{1}$, Castle $J R^{2}$

${ }^{1}$ Department of Biomedical Engineering, Oregon Health and Science University, Portland, OR; ${ }^{2}$ Department of Medicine, Division of Endocrinology, Harold Schnitzer Diabetes Health Center, Oregon Health and Science University, Portland, OR; ${ }^{3}$ Oregon Clinical and Translational Research Institute Biostatistics and Design Program, Oregon Health and Science University, Portland OR; ${ }^{4}$ Department of Medicine, Division of Health Promotion and Sports Medicine, Human Performance Laboratory, Oregon Health and Science University, Portland, OR

\section{Diabetes Obes Metab 2016; 18: 1110-1119}

\section{Background}

With high risks of postexercise hypoglycemia in patients with T1DM, this study examines whether the dual-hormone AP system can reduce exercise-related hypoglycemia.

\section{Methods}

The study recruited 21 adults with T1DM to participate in random order three 22-h experiments: AP with exercise dosing adjustment (APX); AP with no exercise dosing adjustment (APN); and sensor-augmented pump (SAP) therapy. For the exercise sessions, participants exercised at $60 \%$ of their maximum heart rate for 45 minutes. In participants using APX, insulin was decreased and glucagon was increased at the start of the exercise. With SAP therapy, participants adjusted insulin dosing before beginning to exercise. The co-primary outcomes were percentage of time spent in hypoglycemia $(<3.9 \mathrm{mmol} / \mathrm{L})$ and in euglycemia $(3.9-10$ $\mathrm{mmol} / \mathrm{L}$ ) during the physical activity.

\section{Results}

From the start of the exercise to the end of the study, the average time spent in hypoglycemia was $0.3 \%$ [95\% confidence interval - 0.1, 0.7] for APX, 3.1\% [0.8, 5.3] for APN, and $0.8 \%[0.1,1.4]$ for SAP therapy [median (interquartile range)]. A difference of $2.8 \%$ less time spent in hypoglycemia for APX vs. APN was observed $(P=0.001)$. No significant difference was found between APX and SAP therapy. Mean time spent in euglycemia was similar among all three experiments. There were 11 adverse events during the study, in addition to 5 instances of nausea: 2, 2, and 1 for APN, APX and SAP, respectively.

\section{Conclusion}

Adjusting insulin and glucagon exercise doses within a dual-hormone AP resulted in significant reduction in hypoglycemia, similar to adjustments in insulin doses before exercise when SAP therapy was used.

\section{Comments}

Glucagon deficiency plays an important role in the inability of patients with T1DM to cope with exercise, especially when it is unplanned. A study in healthy individuals undergoing moderate exercise revealed reduced insulin levels and doubling of glucagon concentrations, but in subjects with T1DM insulin remained higher with only a partial rise in glucagon, compromising their ability to cope with exercise. Due to its immediate glucose raising effect, glucagon may prevent exercise-induced hypoglycemia (11). This study demonstrated that addition of glucagon while performing exercise can prevent patients from experiencing low blood glucose. Although this study showed this can also be achieved with manual reductions of insulin ahead of time, the dual-hormone system can effectively prevent the need for this type of planning. For future studies, it would be good to compare this system with a singlehormone AP to see how the effectiveness may compare to AP with insulin alone.

\section{Efficacy of single-hormone and dual-hormone artificial pancreas during continuous and interval exercise in adult patients with type 1 diabetes: randomised controlled crossover trial}

Taleb $N^{1,2}$, Emami $A^{1,3}$, Suppere $C^{l}$, Messier $V^{1}$, Legault $L^{4}$, Ladouceur $M^{5}$, Chiasson $J-L,{ }^{5,6}$, Haidar $A^{7,8}$, Rabasa-Lhoret $R^{1,6,9}$

${ }^{1}$ Institut de recherches cliniques de Montréal, Montréal, Québec, Canada; ${ }^{2}$ Division of Biomedical Sciences, Faculty of Medicine, Université de Montréal, Montréal, Québec, Canada; ${ }^{3}$ Division of Experimental Medicine, Department of Medicine, McGill University, Montréal, Québec, Canada; ${ }^{4}$ Montreal Children's Hospital, McGill University Health Centre, Montréal, Québec, Canada: ${ }^{5}$ Centre de Recherche du Centre hospitalier de l'université de Montréal, Montréal, Québec, Canada; ${ }^{6}$ Montreal Diabetes Research Center, Montréal, Québec, Canada; ${ }^{7}$ Department of Biomedical Engineering, Faculty of Medicine, McGill University, Montréal, Québec, Canada; ${ }^{8}$ Division of Endocrinology, Faculty of Medicine, McGill University, Montréal, Québec, Canada; ${ }^{9}$ Nutrition Department, Faculty of Medicine, Université de Montréal, Montréal, Québec, Canada

\section{Diabetologia 2016; 59: 2561-2571}

\section{Background}

A trial was conducted to assess whether the dual-hormone artificial pancreas reduces hypoglycemia versus the singlehormone artificial pancreas across two different types of exercise.

\section{Methods}

An open-label randomized crossover study recruited 17 adults with T1DM to perform two exercise types on an ergocycle: continuous $\left(60 \% \mathrm{VO}_{2}\right.$ peak for $\left.60 \mathrm{~min}\right)$ and interval (2-min alternating periods at $85 \%$ and $50 \% \mathrm{VO}_{2}$ peak for 40 min, with two 10 -min periods at $45 \% \mathrm{VO}_{2}$ peak at the start and end of the session). Exercise was started at 18:00 hours 
and announced $20 \mathrm{~min}$ earlier to the systems. The primary outcome was exercise-induced hypoglycemia requiring treatment.

\section{Results}

Exercise-induced hypoglycemia requiring treatment intervention occurred in four participants $(23.5 \%)$ with singlehormone AP vs. two (11.8\%) interventions with dual-hormone AP $(P=0.5)$ for continuous exercise and six $(40 \%)$ with single-hormone AP vs. one $(6.25 \%)$ for dual-hormone AP $(P=0.07)$ for interval exercise. The median percentage time spent at glucose levels below $4.0 \mathrm{mmol} / \mathrm{L}$ was $11 \%$ vs. $0 \%$ $(P=0.0001)$ and at glucose levels between 4.0 and 10.0 $\mathrm{mmol} / \mathrm{L}$ was $71.4 \%$ vs. $100 \%(P=0.003)$ in favor of dualhormone AP. Higher doses of glucagon were needed during continuous exercise $(0.126 \pm 0.057 \mathrm{mg})$ than during interval exercise $(0.093 \pm 0.068 \mathrm{mg})(P=0.03)$. For all interventions, there with no reported side effects.

\section{Conclusion}

Fewer treated hypoglycemic events occurred with the dual-hormone AP compared with single-hormone AP. In adults with T1DM, the dual-hormone AP outperformed the single-hormone AP in regulating glucose levels during announced exercise.

\section{Comments}

The differences between single- and dual-hormone AP coupled with exercise were clearly demonstrated in this study. This well-planned study took into account standardization of exercise expenditure for each intervention. Based on these results, larger studies should be performed to compare different types of exercise and to see how patients tolerate the interventions in the longer term and in the unsupervised home use setting.

\section{Heart rate informed artificial pancreas system enhances glycemic control during exercise in adolescents with T1DM}

DeBoer $M D^{1,2}$, Cherñavvsky $D R^{1}$, Topchyan $K^{3}$, Kovatchev $B P^{1}$, Francis $G L^{3}$, Breton $M D^{1,4}$

${ }^{1}$ Center for Diabetes Technology, University of Virginia, Charlottesville, VA; ${ }^{2}$ Division of Pediatric Endocrinology, Department of Pediatrics, University of Virginia, Charlottesville, VA; ${ }^{3}$ Division of Endocrinology, Department of Medicine, University of Virginia, Charlottesville, VA; ${ }^{4} \mathrm{Di}$ vision of Pediatric Endocrinology, Department of Pediatrics, Virginia Commonwealth University, Richmond, VA

\section{Pediatric Diabetes 2017; 18: 540-546}

\section{Background}

The variability of insulin needs during exercise is a challenge to AP systems. Therefore, there is a need for an exercise-informed AP that can provide the right amount of insulin based on physiological changes of the body. This study sought to evaluate the safety and performance of using a heart rate (HR) monitor to inform an artificial pancreas (AP) system during exercise among adolescents with type 1 diabetes (T1DM).

\section{Methods}

This was a randomized, crossover trial in adolescents (1318 years of age) with T1DM. Participants were assigned to receive, on separate days, one of the following: (a) the unmodified University of Virginia AP (stdAP), or (b) an AP system connected to a portable heart rate monitor (AP-HR) that triggered an exercise algorithm for blood glucose (BG) control. Subjects participated in a structured exercise regimen. Primary outcome was the number of hypoglycemic episodes.

\section{Results}

Eighteen participants completed the trial. The number of hypoglycemic events during exercise and rest did not differ between visits (0.39 AP-HR vs. 0.50 stdAP). However, time below $70 \mathrm{mg} / \mathrm{dL}$ was lower on AP-HR vs. stdAP $(0.5 \pm 2.1 \%$ vs $7.4 \pm 12.5 \% ; P=0.028)$. Time with $\mathrm{BG}$ within target range $(70-180 \mathrm{mg} / \mathrm{dL})$ was also higher for the AP-HR admission compared with stdAP during the exercise portion and overall ( $96 \%$ vs. $87 \%$ and $77 \%$ vs. $74 \%$ ), but these differences did not demonstrate significance $(P=0.075$ and $\mathrm{p}=0.366)$.

\section{Conclusion}

Heart rate signals can safely and efficiently inform of physical activity in AP systems. Although exercise contributed to hypoglycemia even when using an AP system, informing the system of exercise via the HR monitor improved time in target glucose levels. It did not, however, cause a significant reduction in the total number of hypoglycemic events, which were low in both groups.

\section{Comments}

This study adds additional inputs to AP, which could prove very valuable. This study used a Zypher HxM Bluetooth Heartrate Monitor, which was a chest harness to measure HR data. In the future, HR trackers (such as wristwatch, sensor stickers, etc.) could be integrated with the AP to improve ease of wear. Nevertheless, with the HR-informed AP, hypoglycemic events were not completely avoided, which may be a limitation of singlehormone AP when exercise is detected in real time and insulin dosing is not adjusted beforehand. Although utilizing physiological changes in the body to give more accurate predictions for AP insulin delivery in this study showed good results, there is still room for improvement for future studies where additional signals (such as accelerometry) could be integrated.

\section{Closed-loop insulin delivery for adults with type 1 diabetes undertaking high-intensity interval exercise versus moderate-intensity exercise: a randomized, crossover study}

\section{Jayawardene $D C^{1}$, McAuley $S A^{1,2}$, Horsburgh $J C^{2}$,} Gerche $A L^{3,4}$, Jenkins $A J^{1,2,5}$, Ward $G M^{1,6}$, MacIsaac $R J^{1,2}$, Roberts $T^{2,4}$, Grosman $B^{7}$, Kurtz $N^{7}$, Roy $A^{7}$, O'Neal $D N^{1,2}$ 
${ }^{1}$ Department of Endocrinology and Diabetes, St Vincent's Hospital Melbourne, Melbourne, Australia; ${ }^{2}$ University of Melbourne Department of Medicine, St. Vincent's Hospital, Melbourne, Australia; ${ }^{3}$ Department of Sports Cardiology, Baker Heart and Diabetes Institute, Melbourne, Australia; ${ }^{4}$ Department of Cardiology, St Vincent's Hospital Melbourne, Melbourne, Australia; ${ }^{5}$ NHMRC Clinical Trials Centre, University of Sydney, Sydney, Australia; ${ }^{6}$ Department of Pathology, University of Melbourne, Melbourne, Australia; ${ }^{7}$ Medtronic Diabetes, Northridge, CA

Diabetes Technol Ther 2017; 19: 340-348

\section{Background}

Although previous studies have looked at exercise and closed-loop systems, close studies comparing the types of exercise with regard to closed-loop control have not been conducted. This study specifically compared closed-loop glucose control for people with T1DM undergoing high-intensity interval exercise (HIIE) versus moderate-intensity exercise (MIE).

\section{Methods}

Twelve adults with T1DM established on insulin pumps completed HIIE and MIE stages randomly while using a closed-loop system (MiniMed® 670G and Guardian Sensor 3). Frequent venous samples were collected and tested for glucose, lactate, ketones, insulin, catecholamines, cortisol, growth hormone, and glucagon levels. Plasma glucose $<4.0 \mathrm{mmol} / \mathrm{L}$ for $\geq 15 \mathrm{~min}$, from onset of exercise to $120 \mathrm{~min}$ postexercise, was the primary outcome. Secondary outcomes included continuous glucose monitoring and biochemical parameters.

\section{Results}

All participants completed the study. One participant experienced a drop in plasma glucose to $3.4 \mathrm{mmol} / \mathrm{L}$ following MIE completion; no glucose levels fell below $4.0 \mathrm{mmol} / \mathrm{L}$ for HIIE. No glucose excursions exceeded $15.0 \mathrm{mmol} / \mathrm{L}$ for either type of exercise. Mean plasma glucose $( \pm S D)$ did not differ between stages preexercise, was higher during HIIE than during MIE $(11.3 \pm 0.5 \mathrm{mmol} / \mathrm{L}$ vs. $9.7 \pm 0.6 \mathrm{mmol} / \mathrm{L}$; $P<0.001$ ), and remained higher until $60 \mathrm{~min}$ postexercise. Circulating free insulin remained stable before, during, and after exercise. Significant increases in lactate, catecholamines, and cortisol occurred during HIIE compared with MIE. Ketones increased more with HIIE than MIE postexercise $(P=0.031)$

\section{Conclusion}

Closed-loop glucose control is safe for people undertaking HIIE and MIE. However, the rise in ketones observed with HIIE may represent a challenge for closed-loop systems.

\section{Comments}

This paper reveals that even with the CLC system, different exercise regimens have different impacts on glucose levels. In particular, high intensity exercise may lead to a rise in ketones. Larger studies with longerduration follow-up are needed to see how closed-loop control works with different types of exercise.

\section{TYPE 2 DIABETES}

\section{Closed-loop insulin delivery in inpatients with type 2 diabetes: a randomised, parallel-group trial}

Thabit $H^{1,2}$, Hartnell $S^{2}$, Allen $J M^{1,3}$, Lake $A^{l}$, Wilinska $M E^{1,3}$, Ruan $Y^{1,3}$, Evans $M L^{1,2}$, Coll $A P^{1,2}$, Hovorka $R^{1,3}$

${ }^{1}$ Wellcome Trust-MRC Institute of Metabolic Science, University of Cambridge, Cambridge, UK; ${ }^{2}$ Wolfson Diabetes Endocrine Clinic, Cambridge University Hospitals NHS Foundation Trust, Cambridge, UK; ${ }^{3}$ Department of Paediatrics, University of Cambridge, Cambridge, UK

\section{Lancet Diabetes Endocrinol 2017; 5: 117-124}

\section{Background}

With the emergence of AP systems, clinical studies have tested primarily on participants with type 1 diabetes (T1D). This is one of the first studies to test AP on subjects with type 2 diabetes (T2D) treated with insulin. The goal was to assess the safety and effectiveness of AP compared with standard subcutaneous insulin therapy in patients with T2D in the general ward.

\section{Methods}

This study was an open-label, single-center, parallelgroup, randomized controlled trial including 40 adult T2D patients receiving insulin therapy from general wards at Addenbrooke's Hospital, Cambridge, UK. Participants were randomly assigned to receive either closed-loop insulin delivery without mealtime boluses or conventional subcutaneous insulin delivery. The primary outcome was time glucose levels spent within the target range of 5.6-10.0 $\mathrm{mmol} / \mathrm{L}$ during the 72-h study period.

\section{Results}

The percentage of time glucose levels spent within the target range was significantly higher in the closed-loop group $(59.8 \pm 18.7 \%)$ compared with the control group $(38.1 \pm 16.7 \%)$ $(P=0.0004)$. No participants in either group experienced episodes of severe hypoglycemia or hyperglycemia with ketonemia. During the study, one adverse event of gastrointestinal bleeding occurred unrelated to study devices.

\section{Conclusion}

Closed-loop insulin delivery without mealtime boluses was found to be effective and safe for treatment of adults with T2D who are treated with insulin in the general ward of a hospital.

\section{Comments}

This study was the first to test a closed-loop system for insulin-treated adults with T2D in the general ward. As the closed-loop insulin delivery system has been primarily evaluated in people with $\mathrm{T} 1 \mathrm{D}$, future studies should include people with T2D to test for efficacy in a variety of circumstances. The acceptance of the closedloop system was also evaluated in the study. The majority of participants reported being happy with having 
their glucose levels controlled by an automated system and would recommend closed-loop if their friend or family was in the hospital. It will be important to survey the ward staff as well on their thoughts of closed-loop control for patients with T2D in an inpatient setting, as the addition of more medical devices can affect nurse workload. Future implications include a closed-loop system that functions effectively for everyone affected by diabetes.

\section{PREGNANCY}

\section{Closed-loop insulin delivery during pregnancy in women with type 1 diabetes}

Stewart $Z A^{1}$, Wilinska $M E^{1}$, Hartnell $S^{2}$, Temple $R C^{3}$, Rayman $G^{6}$, Stanley $K P^{4}$, Simmons $D^{2}$, Law $G R^{7}$, Scott $\mathrm{EM}^{7}$, Hovorka $\mathrm{R}^{1}$, Murphy $\mathrm{HR}^{1-3,5}$

${ }^{1}$ Wellcome Trust-Medical Research Council Institute of Metabolic Science, University of Cambridge, Cambridge, $U K ;{ }^{2}$ Wolfson Diabetes and Endocrine Clinic, Cambridge University Hospitals NHS Foundation Trust, Cambridge, UK; ${ }^{3}$ Elsie Bertram Diabetes Centre University of East Anglia, Norwich, UK; ${ }^{4}$ Department of Obstetrics and Gynaecology, Norfolk and Norwich University Hospitals NHS Foundation Trust, University of East Anglia, Norwich, UK; ${ }^{5}$ Norwich Medical School, University of East Anglia, Norwich, UK; ${ }^{6}$ Ipswich Diabetes Centre, Ipswich Hospital NHS Trust, Ipswich, UK; ${ }^{7}$ Division of Epidemiology and Biostatistics, Leeds Institute of Cardiovascular and Metabolic Medicine, University of Leeds, Leeds, UK

N Engl J Med 2016; 375: 644-654

This manuscript is also discussed in the article on Technology and Pregnancy, page S-94.

\section{Background}

Patients with type 1 diabetes have a more difficult time maintaining glycemic control during pregnancy. As previous AP trials have excluded pregnant women with type 1 diabetes, this novel study aimed to provide data on the efficacy, safety, and feasibility of closed-loop systems to improve glycemic control during pregnancy, labor, and delivery.

\section{Methods}

This was a multicenter, open-label, randomized, crossover study comparing 4 weeks of overnight closed-loop therapy with sensor-augmented pump therapy, followed by an extension period of day and night use of a closed-loop system until 48 hours after delivery. The study included 16 pregnant women with type 1 diabetes aged $34.1 \pm 4.6$ years, with a pregnancy of $14 \pm 3.3$ weeks of gestation and a glycated hemoglobin level of $6.8 \pm 0.6 \%$. Fourteen of the participants chose to use the closed-loop system for day and night until delivery. The primary outcome was the percentage of time in which overnight glucose levels were within the target range of $63-140 \mathrm{mg} / \mathrm{dL}$ (3.5 to $7.8 \mathrm{mmol} / \mathrm{L})$.

\section{Results}

During closed-loop therapy, the percentage of time in which overnight glucose levels were in the target range was significantly higher than during sensor-augmented therapy $(74.7 \%$ vs. $59.5 \% ; P=0.002)$. In addition, mean glucose level overnight was significantly lower during closed-loop therapy than during sensor-augmented pump therapy (6.6 vs. 7.4 $\mathrm{mmol} / \mathrm{L}, P=0.009)$. No significant difference was found in the percentage of time in which glucose levels were below the target range (1.3 vs. $1.9 \%$ ), nor in insulin doses or adverseevent rates between closed-loop and sensor-augmented pump therapy. During the extension (up to 14.6 additional weeks), day- and night-phase glucose levels were in the target range $68.7 \%$ of the time, with the mean glucose level being 7.0 $\mathrm{mmol} / \mathrm{L}$. No episodes of severe hypoglycemia occurred during the study.

\section{Conclusion}

The use of overnight closed-loop therapy compared with sensor-augmented pump therapy resulted in better glucose control in pregnant women with type 1 diabetes. The use of day and night closed-loop therapy maintained glycemic control during a high proportion of the time in a period that encompassed antenatal hospital admission, labor, and delivery.

\section{Comments}

Glucose control during pregnancy for women with type 1 diabetes is a significant challenge, but tight glycemic control is needed to prevent congenital anomalies associated with poor glycemic control. Closed-loop control holds promise for many pregnant women with type 1 diabetes. This study showed that closed-loop therapy improved glycemic outcomes significantly for pregnant women throughout the night and in the early hours of the day. Also of note, during a recent follow-up study, $80 \%$ of the participants felt less concerned for their overnight hypoglycemia and that diabetes "did not run their lives" (6). This study shows the potential of AP to improve diabetes care not only for the pregnant mother but also the potential to improve outcomes for the fetus. Longterm studies with much larger numbers of pregnant women using AP are needed to evaluate this hypothesis.

\section{INTRAPERITONEAL INSULIN DELIVERY}

\section{Intraperitoneal insulin delivery provides superior glycemic regulation to subcutaneous insulin delivery in model predictive control-based fully-automated artificial pancreas in patients with type 1 diabetes: a pilot study}

Dassau $E^{1,2}$, Renard $E^{3,4}$, Place $J^{4}$, Farret $A^{3,4}$, Pelletier $M-J^{3}$, Lee $J^{2}$, Huyett $L M^{2}$, Chakrabarty $A^{1}$, Doyle FJ III ${ }^{1,2}$, Zisser $\mathrm{HC}^{2}$

${ }^{1}$ Harvard John A. Paulson School of Engineering and Applied Sciences, Harvard University, Cambridge, MA; ${ }^{2}$ Department of Chemical Engineering, University of California Santa Barbara, Santa Barbara, CA; ${ }^{3}$ Department of Endocrinology, Diabetes, Nutrition and INSERM Clinical 
Investigation Center 1411, University Hospital of Montpellier, Montpellier, France; ${ }^{4}$ Department of Psychology, Institute of Functional Genomics, CNRS UMR5203, INSERM U1191, University of Montpellier, Montpellier, France

Diabetes Obes Metab 2017; 19: 1698-1705

\section{Background}

Intraperitoneal (IP) insulin delivery has the potential to increase insulin action in artificial pancreas systems. As opposed to conventional subcutaneous (SC) insulin delivery, IP better simulates the physiological insulin delivery by providing insulin concentration closer to the hepatic portal system. This study assessed IP and SC insulin delivery in an artificial pancreas.

\section{Methods}

This was an open-label, nonrandomized, sequential study using closed-loop therapy for $24 \mathrm{~h}$ with subcutaneous fastacting insulin analog delivery, and then another admission for $24 \mathrm{~h}$ of closed-loop with intraperitoneal regular insulin delivery. The closed-loop system used in the study was a Zone Model Predictive Control algorithm adjusted for IP/SC insulin clearance. The $24-\mathrm{h}$ treatment period included three unannounced meals with 70,40 , and $70 \mathrm{~g}$ of carbohydrates to assess reaction to meal-related disturbances. Primary outcome was time blood glucose spent within the target range of $80-140 \mathrm{mg} / \mathrm{dL}$.

\section{Results}

Ten adults with type 1 diabetes completed the study. The percentage of time in which glucose levels were within the desired range (80-140 mg/dL) and within a target range (70$180 \mathrm{mg} / \mathrm{dL}$ ) was significantly higher during IP delivery compared with SC delivery $(39.8 \pm 7.6 \%$ vs. $25.6 \pm 13.1 \% ; P=0.03$ and $65.7 \pm 9.2 \%$ vs. $43.9 \pm 14.7 \% ; P=0.001$ respectively). Mean BG was also significantly better with IP regular insulin delivery $(151.0 \pm 11.0$ vs. $190.0 \pm 31.0 ; P=0.004)$.

The improved glycemic control observed during IP delivery was attributed to reduced time spent in hyperglycemia above $180 \mathrm{mg} / \mathrm{dL}$ as well as above $250 \mathrm{mg} / \mathrm{dL}$ compared with SC delivery $(32.4 \pm 8.9 \%$ vs. $53.5 \pm 17.4 \%$ and $5.9 \pm 5.6 \%$ vs. $23.0 \pm 11.3 \%$, respectively) with no increase in the percentage of time spent in hypoglycemia below $70 \mathrm{mg} / \mathrm{dL}$. Significantly higher doses of insulin were delivered during IP delivery compared with SC delivery $(43.7 \pm 0.1$ vs. $32.3 \pm 0.1$ units; $P<0.001)$, likely due to faster insulin clearance.

\section{Conclusion}

Glycemic control with AP delivering IP insulin was found to be better than SC insulin delivery.

\section{Comments}

This study showed IP insulin delivery can improve glucose control over SC when AP is active. One of the greatest challenges of diabetes treatment is to reduce elevated postprandial glucose levels. This is also the main obstacle for a full closed-loop system, mainly due to the delayed onset of the current available insulins.
Therefore, most closed-loop systems are "hybrid," meaning the patient still needs to bolus for meals. The use of the IP route may facilitate insulin action and holds the potential to be a truly full closed-loop system.

Additional study will be important for determining why statistical postprandial improvements in glucose control only occurred for the dinner meal, as faster peak insulin action via IP delivery has the potential to vastly improve the meal response. With improvements to the AP and long-term usage, IP insulin delivery could further improve diabetes care, potentially minimizing the need for prebolusing of meals and improving mean glucose while still minimizing hypoglycemia.

\section{ADDITIONAL SINGLE HORMONE AP STUDIES}

\section{Day-and-night glycaemic control with closed-loop insulin delivery versus conventional insulin pump therapy in free-living adults with well controlled type 1 diabetes: an open-label, randomised, crossover study}

Bally $L^{1,3,4}$, Thabit $H^{1,3}$, Kojzar $H^{5}$, Mader $J K^{5}$, Qerimi-Hyseni $J^{5}$, Hartnell $S^{3}$, Tauschmann $M^{1,2}$, Allen $J M^{1,2}$, Wilinska $M E^{1,2}$, Pieber $T R^{5}$, Evans $M L^{1,3}$, Hovorka $R^{1,2}$

${ }^{1}$ Wellcome Trust-Medical Research Council Institute of Metabolic Science and ${ }^{2}$ Department of Paediatrics, University of Cambridge, Cambridge, UK; ${ }^{3}$ Department of Diabetes \& Endocrinology, Cambridge University Hospitals National Health Service Foundation Trust, Cambridge, UK; ${ }^{4}$ Department of Diabetes, Endocrinology, Clinical Nutrition \& Metabolism, Inselspital, Bern University Hospital, University of Bern, Bern, Switzerland; ${ }^{5}$ Department of Internal Medicine, Division of Endocrinology \& Diabetology, Medical University of Graz, Graz, Austria

Lancet Diabetes Endocrinol 2017; 5: 261-270

\section{Background}

Tight glycemic control was found to be coupled with increased rate of hypoglycemia in people with T1DM. Wellcontrolled subjects may further improve their glycemic control without increased rate of hypoglycemia while using a closed-loop system.

\section{Methods}

This was an open-label, randomized, crossover study. Adults with T1DM and HbA1c below 7.5\% from Addenbrooke's Hospital (Cambridge, UK) and Medical University of Graz (Graz, Austria) were included in the study. Twentynine subjects were randomly assigned to participate in two crossover periods, each including 4 weeks (day and night) of free living conditions. One group was under hybrid closedloop therapy and the second under sensor-augmented pump (SAP) therapy with a washout period of 2-4 weeks between the two interventions. The primary end point was time spent with sensor glucose levels within the target range (3.9-10.0 $\mathrm{mmol} / \mathrm{L}$ ) over the 4-week study intervention period. 


\section{Results}

The percentage of time during which sensor glucose levels were within the target range was increased by $10.5 \%$ during closed-loop delivery compared with usual pump therapy (65.6 $\pm 8.1 \%$ vs. $76.2 \pm 6.4 \%$, respectively; $P<0.001)$, and the time spent in hypoglycemia below $3.5 \mathrm{mmol} / \mathrm{L}$ was reduced by $65 \%$ ([95\% CI 53-74]; $P<0.0001$ ) and below 2.8 mmol/L by $76 \%$ ([59-86]; $P<0.0001)$. No severe adverse events occurred during either of the study arms.

\section{Conclusion}

The use of hybrid closed-loop therapy for day and night glucose control for well-controlled adults with type 1 diabetes under unsupervised, free-living conditions was found to be safe and further improves glucose control with a reduction in the rate of hypoglycemia as well.

\section{Comments}

This study importantly shows that AP can improve glucose control even for those subjects with good baseline glucose control. This population of patients may be the first to adopt closed-loop systems and was less studied in various closed-loop trials. The study did not include subjects with hypoglycemia unawareness, a subset of patients that may also benefit from automated insulin delivery. Another significant finding for this population is that glycemic variability (measured as coefficient of variation and standard deviation) was significantly less in closed-loop during both day and night periods compared with control. The reduced variability can also contribute to the reduced rate of hypoglycemia.

\section{Application of zone model predictive control artificial pancreas during extended use of infusion set and sensor: a randomized crossover-controlled home-use trial}

Forlenza $G P^{1}$, Deshpande $S^{2,3}$, Ly $T T^{4}$, Howsmon $D P^{5}$, Cameron $F^{5}$, Baysal $N^{5}$, Mauritzen $E^{6}$, Marcal $T^{4}$, Towers $L^{1}$, Bequette $W^{5}$, Huyett LM ${ }^{3,7}$, Pinsker JE ${ }^{3}$, Gondhalekar $R^{2,3}$, Doyle FJ III ${ }^{2,3}$, Maahs DM ${ }^{1,4}$, Buckingham $B A^{4}$, Dassau $E^{2,3}$

${ }^{1}$ Barbara Davis Center, University of Colorado Denver, Denver, CO; ${ }^{2}$ Harvard John A. Paulson School of Engineering and Applied Sciences, Harvard University, Cambridge, MA; ${ }^{3}$ William Sansum Diabetes Center, Santa Barbara, CA; ${ }^{4}$ Division of Pediatric Endocrinology and Diabetes, Department of Pediatrics, Stanford University School of Medicine, Stanford, CA; ${ }^{5}$ Department of Chemical and Biological Engineering, Rensselaer Polytechnic Institute, Troy, NY; ${ }^{6}$ Department of Computer Science and Engineering, University of California San Diego, San Diego, CA; 7 Department of Chemical Engineering, University of California Santa Barbara, Santa Barbara, CA

Diabetes Care 2017; 40: 1096-1102

\section{Background}

As AP moves toward becoming the standard of care, it is essential to consider the extended use of insulin infusion sets
(IIS) and continuous glucose monitors (CGMs). An outpatient randomized, crossover study was conducted to test the safety and efficacy of a zone model predictive control (zoneMPC)-based AP system vs. sensor-augmented pump (SAP) therapy while patients wore CGM and IIS for extended periods of time.

\section{Methods}

A total of 19 adults (median age 23 years) with mean HbA1c of $8.0 \pm 1.7 \%$ participated in this crossover, unblinded outpatient study. Participants used a smartphone-based AP system over 2 weeks and compared this with 2 weeks of SAP therapy for 2 weeks, with remote monitoring in both study arms. IIS and CGM failures were provoked via extended wear to 7 and 21 days, respectively.

\section{Results}

The use of AP significantly improved percent time with blood glucose in the range of $70-140 \mathrm{mg} / \mathrm{dL}(48.1 \% \mathrm{vs}$. $39.2 \% ; P=0.016)$ and percent time in $70-180 \mathrm{mg} / \mathrm{dL}(71.6 \%$ vs. $65.2 \% ; P=0.008)$. Median glucose levels were also significantly reduced during closed-loop therapy (141 vs. $153 \mathrm{mg} /$ $\mathrm{dL} ; P=0.036)$ in addition to significant improvement in glycemic variability (SD 52 vs. $55 \mathrm{mg} / \mathrm{dL} ; P=0.044$ ). The percentage of time in hypoglycemia (blood glucose below $70 \mathrm{mg}$ / dL) was significantly reduced while on closed-loop compared with SAP therapy $(1.3 \%$ vs. $2.7 \% ; P=0.001)$. The overnight mean glucose levels were reduced with AP use (140 vs. $158 \mathrm{mg} / \mathrm{dL} ; P=0.02)$. IIS failures $(1.26 \pm 1.44$ vs. $0.78 \pm 0.78$ events; $P=0.13)$ and sensor failures $(0.84 \pm 0.6$ vs. $1.1 \pm 0.73$ events; $P=0.25$ ) were similar between AP and SAP arms.

\section{Conclusion}

Prolonged use of CGM and IIS did not jeopardize glycemic control by zone-MPC. The use of the closed-loop system was found to be safe and significantly improved glycemic control in a home-use environment despite prolonged CGM and IIS wear. As with other technological devices, higher percentage of use (i.e., percent time in closed loop) was associated with better glycemic outcomes. This project represents the first home-use AP study attempting to provoke and detect component failure while successfully maintaining safety and effective glucose control.

\section{Comments}

As with regular use of an insulin pump, it is likely that for real-world use of AP, people with T1DM will wear CGM sensors and IIS longer than the prescribed time. This study shows that even when this occurs, the zone-MPC AP is still very effective and superior to SAP. In fact, with IIS sites used up to 1 week and CGM sensors worn up to 3 weeks, the zone-MPC AP system still performed very well and had superior glycemic results compared with SAP. As this was the first home-use AP study attempting to provoke and detect component failure while successfully maintaining safety and effective glucose control, additional studies should include tests of the durability and extended use accuracy of each part of the AP system to emulate likely real-world use. 
MD-Logic overnight type 1 diabetes control in home settings: a multicentre, multinational, single blind randomized trial

Nimri $R^{1}$, Bratina $B^{2}$, Kordonouri $O^{3}$, Avbelj Stefanija $M^{2}$, Fath $M^{3}$, Biester $T^{3}$, Muller $I^{1}$, Atlas $E^{1}$, Miller $S^{1}$, Fogel $A^{l}$, Phillip $M^{1,4}$, Danne $T^{3}$, Battelino $T^{2,5}$

${ }^{I}$ The Jesse Z and Sara Lea Shafer Institute for Endocrinology and Diabetes, National Center for Childhood Diabetes, Schneider Children's Medical Center of Israel, Petah Tikva, Israel; ${ }^{2}$ Department of Pediatric Endocrinology, Diabetes and Metabolism, University Children's Hospital, Ljubljana, Slovenia; ${ }^{3}$ Diabetes Centre for Children and Adolescents, AUF DER BULT, Kinder und Jugendkrankenhaus, Hannover, Germany; ${ }^{4}$ Sackler Faculty of Medicine, Tel Aviv University, Tel Aviv, Israel; ${ }^{5}$ Faculty of Medicine, University of Ljubljana, Ljubljana, Slovenia

Diabetes Obes Metab 2017; 19: 553-561

\section{Background}

Remote monitoring techniques have been used to provide an extra layer of safety for automated systems. This study evaluated the need for remote monitoring when using the MD-Logic closed-loop system during short-term overnight use at home.

\section{Methods}

Adult patients $(\mathrm{n}=75$; aged $10-54$ years; average A1c $7.8 \pm 0.7 \%$ ) from three clinical sites were enrolled in the study. Patients were randomly assigned to participate in two overnight crossover periods of four nights each divided into closed-loop control or sensor-augmented pump (SAP) therapy in the patient's home. Both study arms were blinded and supervised using a remote-monitoring system. Primary outcomes were time spent with glucose levels $<70 \mathrm{mg} / \mathrm{dL}$ and percentage of nights with mean overnight glucose levels of $90-140 \mathrm{mg} / \mathrm{dL}$.

\section{Results}

Time spent (median [interquartile range]) in hypoglycemia was significantly lower on nights when patients used MDLogic vs. SAP therapy (2.07\% [0-4.78] and 2.6\% [0-10.34], respectively; $P=0.004)$. The percentage of individual nights with a mean overnight glucose level within target range was significantly greater $(75 \%$ [42-75\%] and 50\% [25-75\%]; $P=0.008$ ). Additionally, there was a $28 \%$ increase in time spent within target range $(P=0.001)$, with the same amount of insulin (median [interquartile range] 10.69 units [7.2813.94] and 10.41 units [6.9-14.07]; $P=0.087)$. Calls for hypoglycemia were triggered by remote monitoring at twice the rate during SAP therapy compared with CLC (62 and 29, respectively; $P=0.002$ ).

\section{Conclusion}

The MD-Logic system is safe and effective during overnight use by children, adolescents, and adults with T1DM and therefore provides an effective means of lowering nocturnal hypoglycemia risk.

\section{Comments}

This study encouragingly found that fewer interventions were required for hypoglycemia during closed-loop intervention. In fact, their results showed that use of the MD-Logic algorithm actually eliminated nocturnal hypoglycemia area under the curve at the clinically meaningful level of $60 \mathrm{mg} / \mathrm{dL}$, and that a significant reduction in hypoglycemia was associated with a concomitant significant decrease in all parameters of hyperglycemia, which suggest consideration of the removal of remote monitoring in future studies when AP is active. This is an important finding, as a recent review has shown that some of the improvements in glucose control in prior AP studies may be attributable to remote monitoring being present and subsequent direct supervision from providers as opposed to gains from AP alone (12). This study adds to the significant body of evidence supporting the safety and efficacy of the MD-Logic AP system.

\section{Home use of day-and-night hybrid closed-loop insulin delivery in suboptimally controlled adolescents with type 1 diabetes: a 3-week, free-living, randomized crossover trial}

Tauschmann $M^{1,2}$, Allen $J M^{1,2}$, Wilinska $M E^{1,2}$, Thabit $H^{1}$, Acerini $C L^{2}$, Dunger $D B^{1,2}$, Hovorka $R^{1,2}$

${ }^{1}$ Wellcome Trust-Medical Research Council Institute of Metabolic Science, University of Cambridge, Cambridge, UK; ${ }^{2}$ Department of Paediatrics, University of Cambridge, Cambridge, UK

Diabetes Care 2016; 39: 2019-2025

This manuscript is also discussed in the article on Diabetes Technology and Therapy in the Pediatric Age Group, page S-114.

\section{Background}

Many studies of closed-loop systems have looked at feasibility for home use; therefore, many trials have only tested for day-only or night-only use short term or only in adults. This study assesses the feasibility, safety, and efficacy of dayand-night hybrid closed-loop insulin delivery in adolescents with T1DM under prolonged use in free-living conditions.

\section{Methods}

This was open-label, randomized, crossover study in 12 suboptimally controlled adolescent patients on insulin pump therapy (mean \pm SD age $14.6 \pm 3.1$ years; HbAlc $8.5 \pm 0.7 \%$; duration of diabetes $7.8 \pm 3.5$ years). During two 21 -day periods, hybrid closed-loop insulin delivery was compared with sensor-augmented insulin pump therapy in random order. A model predictive algorithm was used to automatically direct insulin delivery between meals and overnight during the period of closed-loop intervention. Feedback was collected via a follow-up questionnaire that was distributed to participants.

\section{Results}

During closed-loop intervention, sensor glucose was within the target range $(3.9-10 \mathrm{mmol} / \mathrm{L})$ for an increased proportion 
of time compared with sensor-augmented insulin pump therapy $(18.8 \pm 9.8$ percentage points; $P<0.001)$. Mean sensor glucose level decreased by $1.8 \pm 1.3 \mathrm{mmol} / \mathrm{L}(P=0.001)$, and time spent above target also dropped, by $19.3 \pm 11.3 \%$ $(P<0.001)$. The time spent with sensor glucose levels below target range $3.9 \mathrm{mmol} / \mathrm{L}$ was low (median difference 0.4 [interquartile range -2.2 to 1.3 ] percentage points; $P=0.33$ ) and remained comparable between interventions. Glucose control improved during closed-loop therapy. This added control was associated with increased total daily insulin dose (53.5 [39.572.1] vs. 51.5 [37.6-64.3] units/day; $P=0.006)$ and increased variability of basal insulin delivery $(P<0.001)$. The follow-up survey revealed positive patient attitudes and experience with the closed-loop system.

\section{Conclusion}

In suboptimally controlled adolescents with T1DM, freeliving home use of day-and-night closed-loop therapy is safe, feasible, and provides improved glucose control without an increase in hypoglycemia risk.

\section{Comments}

This study importantly showed that AP can improve glucose control in the adolescent population, many of whom have higher $\mathrm{HbA} 1 \mathrm{c}$ than adults. This is a very important finding, as adolescents with suboptimal glucose control are not always adherent with diabetes care and stand the most to gain from AP as shown in this study. The study was of relatively short duration ( 3 weeks in each arm) but proves the safety and feasibility of using the system in the target population. Future long-term trials will help determine the efficacy of the system in this challenging population and will determine if these adolescents are able to use the AP system long enough to see significant benefit to their HbA1c.

\section{Summary}

This year's articles showed the true potential of AP in the outpatient setting. The next step is to take the systems studied and subject them to rigorous randomized controlled outpatient home use trials. As shown in a recent systematic review and meta-analysis, there remain a number of unanswered questions, particularly in studies with remote monitoring that may lead to improved reporting of results (12). Future long-term studies will prove the efficacy of single- and dual-hormone systems, and it is only through rigorous, structured controlled trials in the anticipated real-world home use setting (i.e., without remote monitoring) that investigators will be able to improve AP devices to optimize outcomes. The greatest breakthrough and important milestone in the pathway of implementation of closed-loop systems to regular diabetes treatment was recently made by the FDA approval of the first Medtronic hybrid closed-loop system. Ultimately, patients with diabetes will benefit greatly from the number of systems under development by commercial entities.

\section{Author Disclosure Statement}

R.N. has received consulting fees from NovoNordisk and speaker's bureau from NovoNordisk, Pfizer, Sanofi, and Eli Lilly; has received research support from Medtronic and Dexcom; shareholder and has an active position in DreaMed Diabetes.

E.D. has received consulting fees from Animas and Insulet; has received research support from Dexcom, Animas, Insulet, Roche, Tandem, Lifescan, and Xeris; and receives royalty payments on intellectual property related to closedloop algorithms.

J.E.P. has conducted research sponsored by Tandem, Insulet, and Bigfoot and has received research support to his institution from Insulet, Animas, Lifescan, Roche, and Dexcom.

P.A. reports no competing interests.

\section{References}

1. Huyett LM, Ly TT, Forlenza GP, et al. Outpatient closedloop control with unannounced moderate exercise in adolescents using zone model predictive control. Diabetes Technol Ther 2017; 19: 331-339.

2. Dassau E, Renard E, Place J, et al. Intraperitoneal insulin delivery provides superior glycemic regulation to subcutaneous insulin delivery in model predictive control-based fully-automated artificial pancreas in patients with type 1 diabetes: a pilot study. Diabetes Obes Metab 2017; 19: 1698-1705.

3. Kitagawa H, Yatabe T, Namikawa T, Munekage M, Hanazaki K. Postoperative closed-loop glycemic control using an artificial pancreas in patients after esophagectomy. Anticancer Res 2016; 36: 4063-4067.

4. Mita N, Kawahito S, Soga T, et al. Strict blood glucose control by an artificial endocrine pancreas during hepatectomy may prevent postoperative acute kidney injury. J Artif Organs 2017; 20: 76-83.

5. Namikawa T, Munekage M, Kitagawa H, et al. Comparison between a novel and conventional artificial pancreas for perioperative glycemic control using a closed-loop system. J Artif Organs 2017; 20: 84-90.

6. Farrington C, Stewart ZA, Barnard K, Hovorka R, Murphy HR. Experiences of closed-loop insulin delivery among pregnant women with Type 1 diabetes. Diabet Med 2017; 34: 1461-1469.

7. Sharifi A, De Bock MI, Jayawardene D, et al. Glycemia, treatment satisfaction, cognition, and sleep quality in adults and adolescents with type 1 diabetes when using a closed-loop system overnight versus sensor-augmented pump with low-glucose suspend function: a randomized crossover study. Diabetes Technol Ther 2016; 18: 772783.

8. Troncone A, Bonfanti R, Iafusco D, et al. Evaluating the experience of children with type 1 diabetes and their parents taking part in an artificial pancreas clinical trial over multiple days in a diabetes camp setting. Diabetes Care 2016; 39: 2158-2164.

9. Haidar A, Legault L, Matteau-Pelletier L, et al. Outpatient overnight glucose control with dual-hormone artificial pancreas, single-hormone artificial pancreas, or conventional insulin pump therapy in children and adolescents with type 1 diabetes: an open-label, randomised controlled trial. Lancet Diabetes Endocrinol 2015; 3: 595-604. 
10. El-Khatib FH, Balliro C, Hillard MA, et al. Home use of a bihormonal bionic pancreas versus insulin pump therapy in adults with type 1 diabetes: a multicentre randomised crossover trial. Lancet 2016; 389: 369-380.

11. Kudva YC, Carter RE, Cobelli C, Basu R, Basu A. Closedloop artificial pancreas systems: physiological input to enhance next-generation devices. Diabetes Care 2014; 37: 1184-1190.

12. Weisman A, Bai J-W, Cardinez M, Kramer CK, Perkins BA. Effect of artificial pancreas systems on glycaemic control in patients with type 1 diabetes: a systematic review and metaanalysis of outpatient randomised controlled trials. Lancet Diabetes Endocrinol 2017; 5: 501-512. 